Supporting Information

\title{
2D mechano-thermoelectric heterojunctions for self-powered strain sensors
}

Ying-Yu Wang $\ddagger^{1,2}$, Ding-Rui Chen $t^{3,4}$, Jen-Kai Wu ${ }^{1,2}$, Tian-Hsin Wang ${ }^{5}$, Chiashain Chuang ${ }^{5}$, Ssu-Yen Huang ${ }^{2}$, Wen-Pin Hsieh ${ }^{6}$, Mario Hofmann ${ }^{2}$, Yuan-Huei Chang ${ }^{2}$, Ya-Ping Hsieh*1

${ }^{1}$ Institute of Atomic and Molecular Sciences, Academia Sinica, 10617, Taiwan

${ }^{2}$ Department of Physics, National Taiwan University, Taipei, 10617, Taiwan

${ }^{3}$ International Graduate Program of Molecular Science and Technology, National Taiwan University, Taipei, 10617, Taiwan

${ }^{4}$ Molecular Science and Technology Program, Taiwan International Graduate Program, Academia Sinica, 10617, Taiwan

${ }^{5}$ Department of Electronic Engineering, Chung Yuan Christian University, Taoyuan, 32023, Taiwan

${ }^{6}$ Institute of Earth Science, Academia Sinica, Taipei, 11529, Taiwan

$\$$ These authors contributed equally to this work.

*Corresponding author Email: yphsieh@gate.sinica.edu.tw 


\section{Materials and Methods}

Graphene growth

Graphene was grown on copper foil by chemical vapor deposition as previously reported ${ }^{1}$. Then copper foil was etched by $5 \%$ ammonium persulfate solution, and graphene floated on deionized water over night. Afterwards the graphene was transferred to thermally oxidized silicon wafer $(300 \mathrm{~nm} \mathrm{SiO} 2 / \mathrm{Si})$ and annealed at $65^{\circ} \mathrm{C}$ for 1 hour.

\section{$\underline{\text { SnSe2/graphene growth }}$}

$\mathrm{SnSe}_{2}$ was grown on thus produced graphene/ $\mathrm{SiO}_{2} / \mathrm{Si}\left(\mathrm{G} / \mathrm{SiO}_{2} / \mathrm{Si}\right)$ substrate by a CVD method ${ }^{2}$. About $1.0 \mathrm{mg}$ of $99.999 \% \mathrm{SnI}_{2}$ granules and $2.0 \mathrm{mg}$ of $99.5 \%$ Se powder were put on an alumina boat, and the $\mathrm{G} / \mathrm{SiO} 2 / \mathrm{Si}$ substrate was placed $32 \mathrm{~cm}$ downstream on another alumina boat. Then, the alumina boat was inserted into the quartz tube in the furnace. The source and substrate were heated to $600{ }^{\circ} \mathrm{C}$ and $100{ }^{\circ} \mathrm{C}$, respectively, for the growth of $\mathrm{Sn} \neg \mathrm{Se}_{2}$. The growth was carried out for 20 minutes, and then the furnace was allowed to cool down naturally to room temperature.

\section{Characterization}

\section{Material characterization}

Raman spectroscopy was conducted in a home-built Micro-Raman system with an excitation energy of $532 \mathrm{~nm}$. Raman suggests that the quality of graphene remains high after CVD synthesis of $\mathrm{SnSe} 2$ (Figure S1).The stoichiometric composition of the $\mathrm{SnSe} 2$ was examined with XPS (Figure S2). Besides, the elemental analysis of the $\mathrm{SnSe} 2 /$ graphene was performed using EDS (Figure S3)..

Transmission electron microscopy was carried out in a JEOL JEM-2100F. The morphology of $\mathrm{Sn} \neg \mathrm{Se}_{2}$ on graphene was examined using AFM (Nanosurf FlexAFM) and SEM (Phenom ProX). Then Electrical transport properties were measured on photolithographically defined contacts (5 nm titanium and $100 \mathrm{~nm}$ gold electrodes) using a Keysight B2912A source meter.

The thermal conductance of graphene and $\mathrm{SnSe}_{2}$ /graphene heterojunctions were determined by characterizing the laser-power-dependence of the temperature-induced Raman peak shift ${ }^{3}$ (Figure S5). For this purpose, both materials were transferred onto TEM grids with 30um openings. The interface thermal conductance $\mathrm{G}\left(\equiv \kappa_{d^{-1}}^{-1}\right.$, where $\mathrm{d}$ is the thickness of thermoelectric material) was examined using a home-built TDTR system ${ }^{4-6}$ (Figure S6).

\section{Calculations}

Finite element simulations

Strain localization was computed in a two-dimensional discontinuous film whose grain structure was modeled after SEM images using COMSOL Multiphysics. Each grain was assigned a high elastic modulus corresponding to the combined Youngs modulus of graphene and $\mathrm{SnSe}_{2}$. The boundaries were assumed to only contain the elastic modulus of graphene. The film was subsequently deformed under a constant force that yielded an extension of $0.5 \%$ and the van-Mises stress was extracted.

The temperature distribution within the sensor device was simulated using tabulated values for materials and measured dimensions from Fig. 5(a)). A structure temperature of $40^{\circ} \mathrm{C}$ was assumed based on reported values and an air temperature of $25^{\circ} \mathrm{C}$ was assumed. An airflow speed of $1 \mathrm{~m} / \mathrm{s}$ was employed to calculate convective heat dissipation. 
Estimating interfacial adhesion energy

We estimate the strain energy areal density of graphene that is subjected to a strain of $1 \%$, which corresponds to our experimental observation.

$$
u=\frac{1}{2} E_{2 D} \varepsilon^{2}
$$

, where E2D is the 2D Young's modulus of graphene which has been reported to be $330 \mathrm{~N} / \mathrm{m}$. ${ }^{7}$ Given the areal density of carbon atoms in graphene, we calculate the strain energy per carbon atom to be $260 \mathrm{meV}$ which is much higher than the strength of a van-der-Waals bond. ${ }^{8}$

\section{Comparison of direct and indirectly measured ZT values}

The ZT values presented were obtained by Harman measurements which consider carrier conduction and heat conduction in the 2D material/electrode system. Indirect measurements that consider materials' resistivity, thermal conductivity, and Seebeck coefficient usually do not account for such contact effects. To compare the two metrics, we first measure the resistance of $\mathrm{SnSe}_{2}$ including contact resistance contributions. We find that the apparent resistivity is $4.2 \times 10^{-7} \Omega m$ which is comparable to the intrinsic resistivity due to the high channel resistance of the employed macroscopic device. The importance of contacts is apparent, however, in the apparent thermal conductivity. We measure the thermal conductivity of $\mathrm{SnSe} 2$ in contact with a metal film using time-resolved thermoreflectance measurements (Figure S6). We find a significantly decreased thermal conductivity of $0.14 \mathrm{~W} / \mathrm{mK}$ which indicates the large contact heat resistance. Using these values and the measured Seebeck coefficient $(-22.31 \mu \mathrm{V} / \mathrm{K})$, we obtain a $\mathrm{ZT}$ of 2.5 at room temperature, which is comparable to the directly measured performance. 


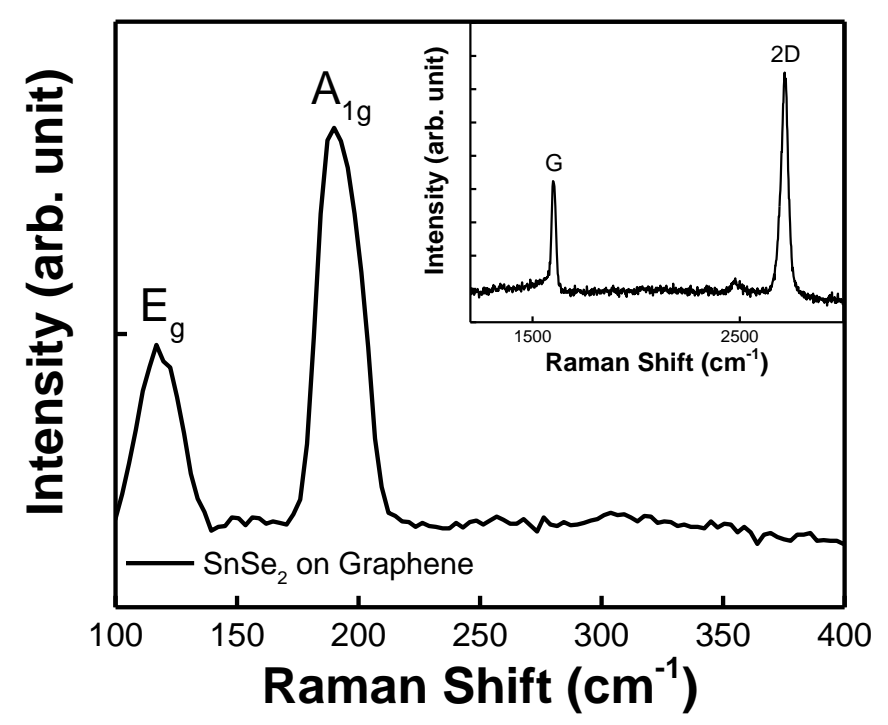

\section{Figure S1.}

Raman spectra of $\mathrm{SnSe}_{2}$ /graphene showing two sharp peaks in the low energy region corresponding to the $\mathrm{E}_{\mathrm{g}}$ and $\mathrm{A}_{\mathrm{lg}}$ modes, at $118.2 \mathrm{~cm}^{-1}$ and $189.9 \mathrm{~cm}^{-1}$, respectively, which are characteristic for $\mathrm{SnSe}_{2}$ thin films ${ }^{2}$. (inset) The presence of graphene can be inferred from the $\mathrm{G}$ and 2D bands at $1587 \mathrm{~cm}^{-1}$ and $2682 \mathrm{~cm}^{-1}$. The absence of the D-band at $1350 \mathrm{~cm}^{-1}$ suggests that the quality of graphene remains high after graphene-assisted $\mathrm{SnSe}_{2}$ growth.
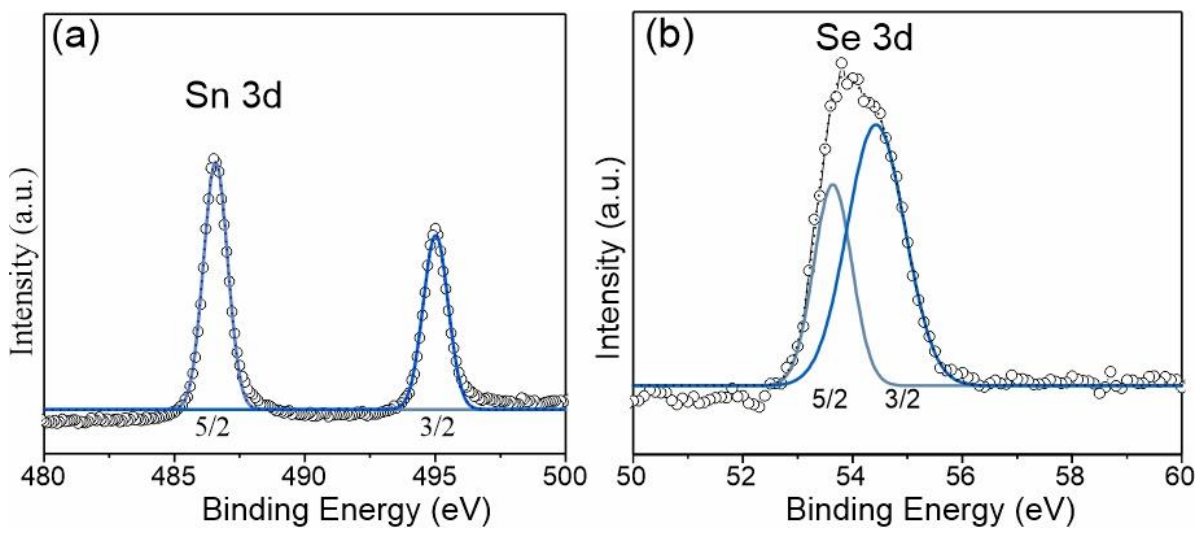

\section{Figure S2.}

XPS spectra of (a) Sn 3d core levels revealing two spin-orbit doublet components with peak position at about $486.6 \mathrm{eV}\left(\mathrm{Sn} 3 \mathrm{~d}_{5 / 2}\right)$ and $495.0 \mathrm{eV}\left(\mathrm{Sn} 3 \mathrm{~d}_{3 / 2}\right)$ which correspond to $\mathrm{Sn}$ (IV) state $^{9}$. (b) Se $3 \mathrm{~d}$ spectra consisting of two peaks, $53.6 \mathrm{eV}$ and $54.4 \mathrm{eV}$ which correspond to Se $3 \mathrm{~d}_{5 / 2}$ and $\mathrm{Se} 3 \mathrm{~d}_{3 / 2}$, respectively. The peak separations for Se and $\mathrm{Sn}$ are $\Delta=8.4 \mathrm{eV}$ and $\Delta=0.80$ $\mathrm{eV}$, respectively, which agrees with previous reports ${ }^{10}$. The ratio of the atomic percentage of $\mathrm{Se}$ and $\mathrm{Sn}$ is close to 2 . 


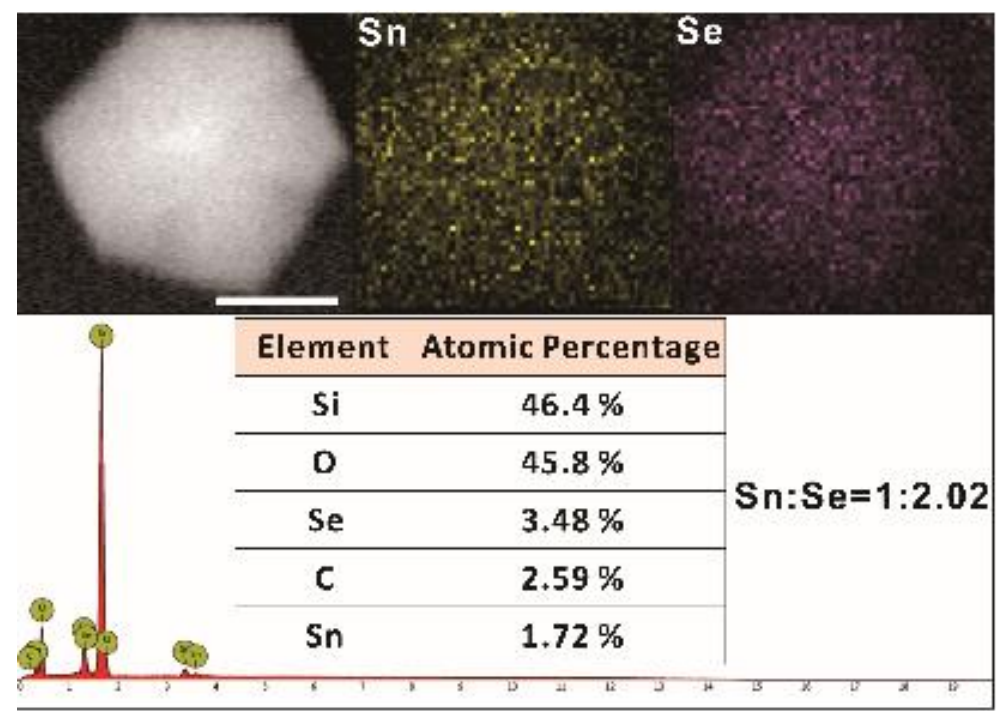

\section{Figure S3.}

Elemental mapping of $\mathrm{SnSe}_{2}$ flake (upper) and the EDS spectrum of $\mathrm{SnSe}_{2}$ grown on Graphene supported on $\mathrm{SiO}_{2} / \mathrm{Si}$ (lower) with indication of occurring elements. Tin and selenium are homogeneously distributed within the nano-flakes, and the ratio of atomic percentage of Se and $\mathrm{Sn}$ is 2.02 which is consistent with the analysis of XPS. The results suggest that our sample possesses pure $\mathrm{SnSe} 2$ grown on $\mathrm{G} / \mathrm{SiO}_{2} / \mathrm{Si}$ substrate without impurity.

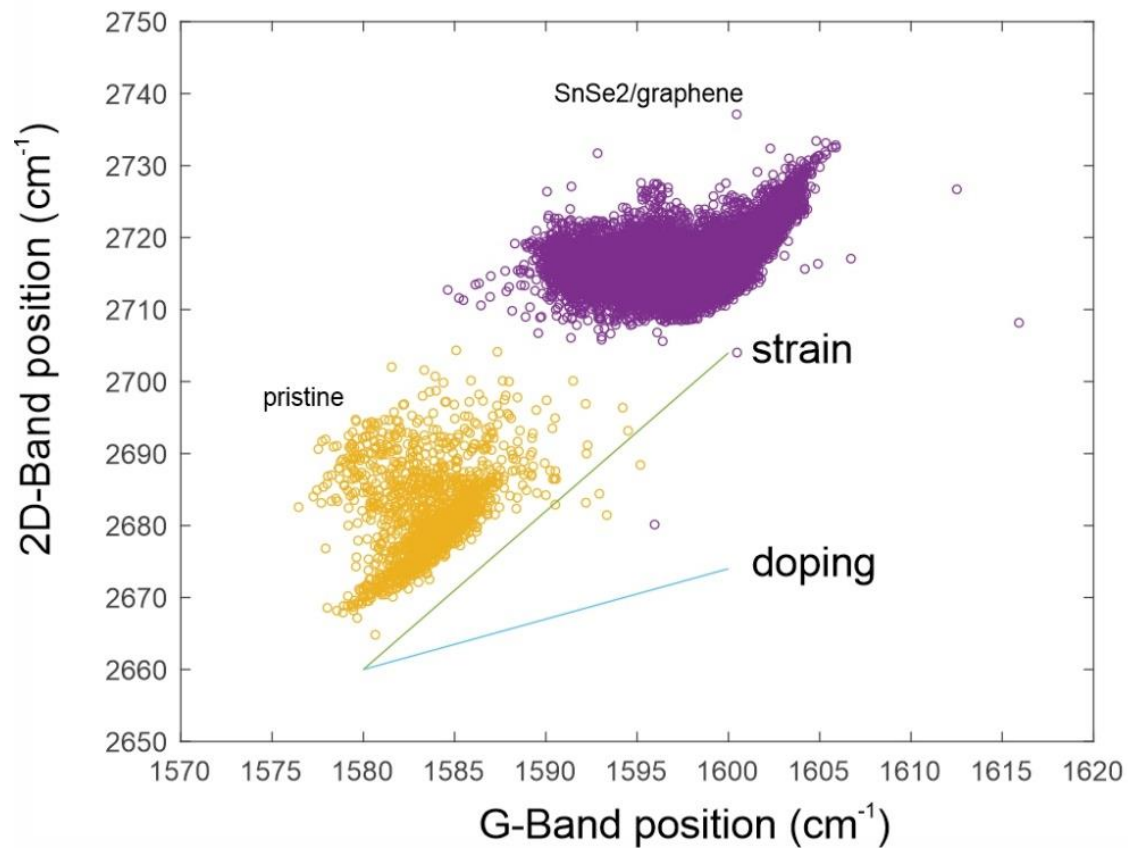

\section{Figure S4.}

Scaling analysis: Distribution of G-band and 2D position for spectra taken from pristine and $\mathrm{SnSe}$ /graphene samples, solid lines represent predictions for graphene samples that only exhibit strain or doping. A basis transformation can be employed to identify charge transfer and strain for each spectrum ${ }^{11}$. 

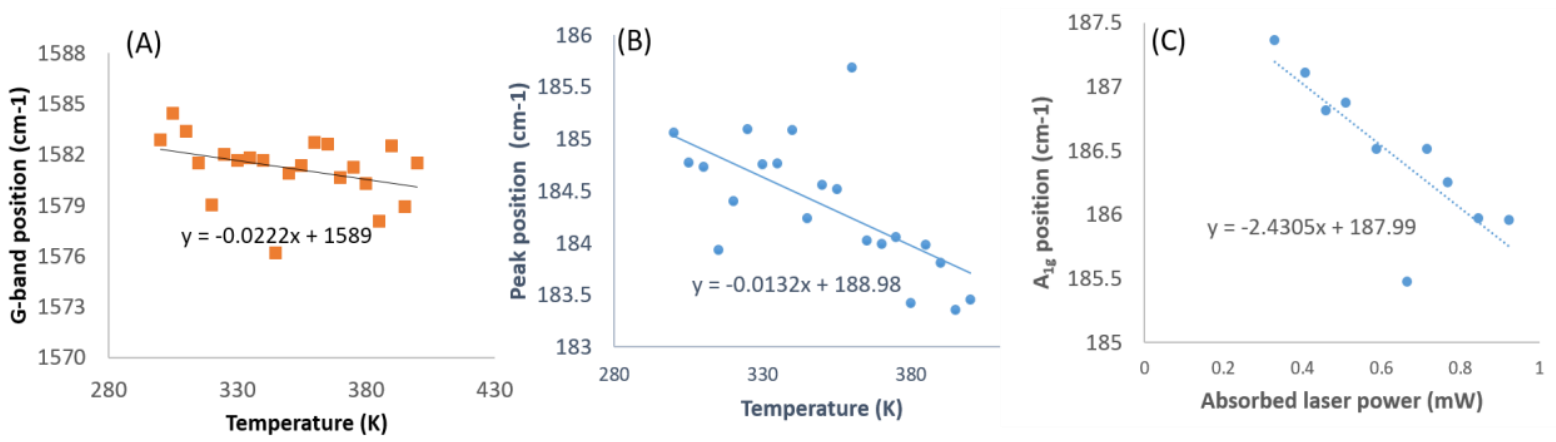

\section{Figure S5.}

Additional Characterization of thermal conductance: (A) Temperature dependence of G-Band position in $\mathrm{SnSe}_{2} /$ graphene with temperature whose slope agrees with previous reports ${ }^{3}$. (B) Temperature calibration of $\mathrm{SnSe}_{2} \mathrm{~A}_{1 \mathrm{~g}}$ peak position exhibiting a slope that is comparable to theoretical predictions ${ }^{12}$. (C) Laser-power dependent $\mathrm{A}_{1 \mathrm{~g}}$ position. With the thickness extracted from AFM, the thermal conductivity can be calculated from G-Band and A1g which yield values of $28.4 \mathrm{~W} / \mathrm{mK}$ and $29.9 \mathrm{~W} / \mathrm{mK}$ for $\mathrm{SnSe}_{2} /$ graphene, respectively.

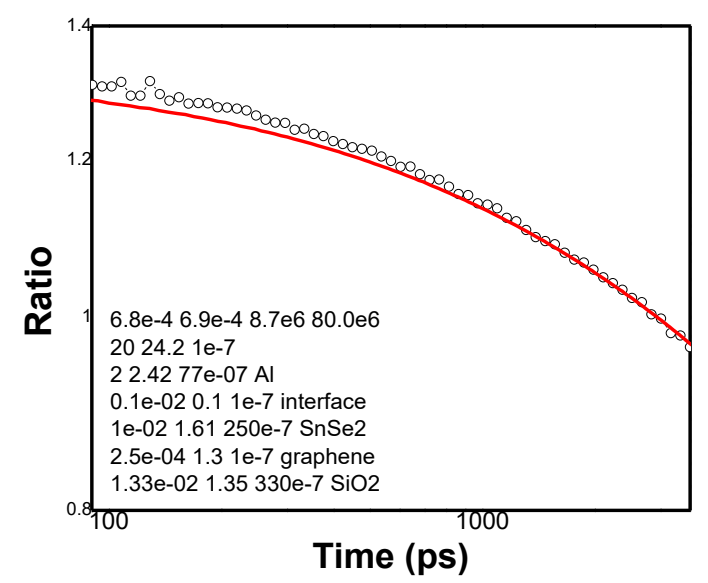

\section{Figure S6.}

Time-resolved thermoreflectance spectrum for $\mathrm{SnSe}_{2}$ /graphene on $\mathrm{SiO}_{2}$ with $\mathrm{Al}$ reflection layer, the solid line represents a fit according to the heat conductivity, index of refraction, and thickness for each layer as determined in the inset. We extract an out-of-plane thermal conductivity of $0.2 \mathrm{~W} / \mathrm{mK}$ indicating the large value of interfacial heat resistance. 


\begin{tabular}{|c|c|c|}
\hline 2D materials & ZT@Temperature & Reference \\
\hline Graphene & $\mathrm{ZT}=0.002 @ 300 \mathrm{~K}$ & 13 \\
\hline Black phosphorus & $\mathrm{ZT}=1.1 @ 300 \mathrm{~K}$ & 14 \\
\hline p-type $\mathrm{MoS}_{2}$ & $\mathrm{ZT}=0.18 @ 1000 \mathrm{~K}$ & 15 \\
\hline 2D tellurium & $\mathrm{ZT}=0.63 @ 300 \mathrm{~K}$ & 16 \\
\hline 2D heterojunctions & ZT@Temperature & Reference \\
\hline $\begin{array}{l}\text { graphene/lanthanum } \\
\text { strontium titanium oxide }\end{array}$ & $\mathrm{ZT}=0.42 @ 300 \mathrm{~K}$ & 17 \\
\hline $\mathrm{Bi}_{2} \mathrm{Se}_{3}$ /graphene & $\mathrm{ZT}=1.03 @ 300 \mathrm{~K}$ & 18 \\
\hline $\mathrm{Bi}_{2} \mathrm{Te}_{3} /$ graphene & $\mathrm{ZT}=0.55 @ 425 \mathrm{~K}$ & 19 \\
\hline $\mathrm{SnS}_{2} / \mathrm{Bi}_{2} \mathrm{Te}_{2.7} \mathrm{Se}_{0.3}$ & $\mathrm{ZT}=0.93 @ 450 \mathrm{~K}$ & 20 \\
\hline
\end{tabular}

Table S1.

Comparison of thermoelectric performance to other 2D materials and heterojunctions. 


\section{References}

1. Hsieh, Y.-P.; Hofmann, M.; Kong, J., Promoter-assisted chemical vapor deposition of graphene. Carbon 2014, 67, 417-423.

2. Zhou, X.; Gan, L.; Tian, W.; Zhang, Q.; Jin, S.; Li, H.; Bando, Y.; Golberg, D.;

Zhai, T., Ultrathin SnSe2 Flakes Grown by Chemical Vapor Deposition for High-

Performance Photodetectors. Adv. Mater. 2015, 27 (48), 8035-8041.

3. Malekpour, H.; Balandin, A. A., Raman-based technique for measuring thermal conductivity of graphene and related materials. J. Raman Spectrosc. 2018, 49 (1), 106-120.

4. Costescu, R. M.; Wall, M. A.; Cahill, D. G., Thermal conductance of epitaxial interfaces. Phys. Rev. B 2003, 67 (5), 054302.

5. Lyeo, H.-K.; Cahill, D. G., Thermal conductance of interfaces between highly dissimilar materials. Phys. Rev. B 2006, 73 (14), 144301.

6. Juang, Z.-Y.; Tseng, C.-C.; Shi, Y.; Hsieh, W.-P.; Ryuzaki, S.; Saito, N.; Hsiung,

C.-E.; Chang, W.-H.; Hernandez, Y.; Han, Y.; Tamada, K.; Li, L.-J., Graphene-Au

nanoparticle based vertical heterostructures: A novel route towards high-ZT Thermoelectric devices. Nano Energy 2017, 38, 385-391.

7. Liu, K.; Wu, J. Q., Mechanical properties of two-dimensional materials and heterostructures. J Mater Res 2016, 31 (7), 832-844.

8. Ahn, G. H.; Amani, M.; Rasool, H.; Lien, D. H.; Mastandrea, J. P.; Ager, J. W.; Dubey, M.; Chrzan, D. C.; Minor, A. M.; Javey, A., Strain-engineered growth of twodimensional materials. Nat Commun 2017, 8.

9. $\quad$ Zhang, Y.; Shi, Y.; Wu, M.; Zhang, K.; Man, B.; Liu, M., Synthesis and SurfaceEnhanced Raman Scattering of Ultrathin SnSe2 Nanoflakes by Chemical Vapor Deposition. Nanomaterials 2018, 8 (7).

10. Saha, S.; Banik, A.; Biswas, K., Few-Layer Nanosheets of n-Type SnSe2. Chem. Eur. J. 2016, 22 (44), 15634-15638.

11. Lee, J. E.; Ahn, G.; Shim, J.; Lee, Y. S.; Ryu, S., Optical separation of mechanical strain from charge doping in graphene. Nat. Commun. 2012, 3 (1), 1024.

12. An, B.; Ma, Y.; Zhang, G.; You, C.; Zhang, Y., Controlled synthesis of few-layer SnSe2 by chemical vapor deposition. RSC Advances 2020, 10 (69), 42157-42163.

13. Anno, Y.; Imakita, Y.; Takei, K.; Akita, S.; Arie, T., Enhancement of graphene thermoelectric performance through defect engineering. 2D Mater. 2017, 4 (2), 025019. 14. Zhang, J.; Liu, H. J.; Cheng, L.; Wei, J.; Liang, J. H.; Fan, D. D.; Jiang, P. H.; Sun, L.; Shi, J., High thermoelectric performance can be achieved in black phosphorus. Journal of Materials Chemistry C 2016, 4 (5), 991-998.

15. Kong, S.; Wu, T.; Zhuang, W.; Jiang, P.; Bao, X., Realizing p-Type MoS2 with Enhanced Thermoelectric Performance by Embedding VMo2S4 Nanoinclusions. J. Phys. Chem. B 2018, 122 (2), 713-720.

16. Qiu, G.; Huang, S.; Segovia, M.; Venuthurumilli, P. K.; Wang, Y.; Wu, W.; Xu, X.; Ye, P. D., Thermoelectric Performance of 2D Tellurium with Accumulation Contacts. Nano Lett. 2019, 19 (3), 1955-1962.

17. Lin, Y.; Norman, C.; Srivastava, D.; Azough, F.; Wang, L.; Robbins, M.;

Simpson, K.; Freer, R.; Kinloch, I. A., Thermoelectric Power Generation from Lanthanum Strontium Titanium Oxide at Room Temperature through the Addition of Graphene. ACS Appl. Mater. Interfaces 2015, 7 (29), 15898-15908.

18. Wu, J.-K.; Hofmann, M.; Hsieh, W.-P.; Chen, S.-H.; Yen, Z.-L.; Chiu, S.-K.; Luo, Y.-R.; Chiang, C.-C.; Huang, S.-Y.; Chang, Y.-H.; Hsieh, Y.-P., Enhancing Thermoelectric Properties of 2D Bi2Se3 by 1D Texturing with Graphene. ACS Appl. Energy Mater. 2019, 2 (12), 8411-8415. 
19. Li, S.; Fan, T.; Liu, X.; Liu, F.; Meng, H.; Liu, Y.; Pan, F., Graphene Quantum Dots Embedded in Bi2Te3 Nanosheets To Enhance Thermoelectric Performance. ACS Appl. Mater. Interfaces 2017, 9 (4), 3677-3685.

20. Li, S.; Liu, X.; Liu, Y.; Liu, F.; Luo, J.; Pan, F., Optimized hetero-interfaces by tuning 2D SnS2 thickness in $\mathrm{Bi} 2 \mathrm{Te} 2.7 \mathrm{Se} 0.3 / \mathrm{SnS} 2$ nanocomposites to enhance thermoelectric performance. Nano Energy 2017, 39, 297-305. 